\title{
The upstream Variable Number Tandem Repeat polymorphism of the monoamine oxidase type A gene influences trigeminal pain-related evoked responses
}

Cherubino Di Lorenzo, ${ }^{1}$ Andrea Daverio, ${ }^{2,3,4}$ Patrizio Pasqualetti, ${ }^{5,6}$ Gianluca Coppola, ${ }^{7}$ Ioannis Giannoudas, ${ }^{2,3,4}$ Ylenia Barone,,$^{3,4}$ Gaetano S. Grieco, ${ }^{8}$ Cinzia Niolu, ${ }^{3,4}$ Esterina Pascale, ${ }^{9}$ Filippo M. Santorelli, ${ }^{10}$ Ferdinando Nicoletti, ${ }^{11,12}$ Francesco Pierelli, ${ }^{11}$ Alberto Siracusano, ${ }^{2,3,4}$ Stefano Seri ${ }^{13}$ and Giorgio Di Lorenzo ${ }^{2,3}$ ${ }^{1}$ Don Carlo Gnocchi Onlus Foundation, Via Maresciallo Caviglia, 30-00135, Rome, Italy

${ }^{2}$ Laboratory of Psychophysiology, Department of Systems Medicine, University of Rome "Tor Vergata", Rome, Italy

${ }^{3}$ Chair of Psychiatry, Department of Systems Medicine, University of Rome "Tor Vergata", Rome, Italy

${ }^{4}$ Psychiatric Clinic, Fondazione Policlinico "Tor Vergata", Rome, Italy

${ }^{5}$ Service of Medical Statistics and Information Technology, Fatebenefratelli Association for Research (AFaR), Rome, Italy

${ }^{6}$ Epidemiology and Biostatistics, Casa di cura San Raffaele-Cassino, Cassino, Italy

${ }^{7}$ Department of Neurophysiology of Vision and Neurophthalmology, G.B. Bietti Eye Foundation-IRCCS, Rome, Italy

${ }^{8}$ Laboratory of Neurogenetics, IRCCS Neurological Institute "C. Mondino", Pavia, Italy

${ }^{9}$ Department of Medico-Surgical Sciences and Biotechnologies, Sapienza University of Rome, Rome, Italy

${ }^{10}$ Molecular Medicine \& Neurodegenerative Diseases, IRCCS Fondazione Stella Maris, Pisa, Italy

${ }^{11}$ IRCCS-Neuromed, Pozzilli, IS, Italy

${ }^{12}$ Department of Pharmacology, Sapienza University of Rome, Rome, Italy

${ }^{13}$ School of Life and Health Sciences, Aston Brain Centre, Aston University, Birmingham, UK

Keywords: habituation, human, monoamine, neural plasticity, pain-related evoked potential, sensitization

\begin{abstract}
Monoamines have an important role in neural plasticity, a key factor in cortical pain processing that promotes changes in neuronal network connectivity. Monoamine oxidase type A (MAOA) is an enzyme that, due to its modulating role in monoaminergic activity, could play a role in cortical pain processing. The X-linked MAOA gene is characterized by an allelic variant of length, the MAOA upstream Variable Number Tandem Repeat (MAOA-uVNTR) region polymorphism. Two allelic variants of this gene are known, the high-activity MAOA (HAM) and low-activity MAOA (LAM). We investigated the role of MAOA-uVNTR in cortical pain processing in a group of healthy individuals measured by the trigeminal electric pain-related evoked potential (tPREP) elicited by repeated painful stimulation. A group of healthy volunteers was genotyped to detect MAOA-UVNTR polymorphism. Electrical tPREPs were recorded by stimulating the right supraorbital nerve with a concentric electrode. The N2 and P2 component amplitude and latency as well as the N2-P2 inter-peak amplitude were measured. The recording was divided into three blocks, each containing 10 consecutive stimuli and the N2-P2 amplitude was compared between blocks. Of the 67 volunteers, 37 were HAM and 30 were LAM. HAM subjects differed from LAM subjects in terms of amplitude of the grand-averaged and first-block N2-P2 responses (HAM>LAM). The N2-P2 amplitude decreased between the first and third block in HAM subjects but not LAM subjects. The MAOA-UVNTR polymorphism seemed to influence the brain response in a repeated tPREP paradigm and suggested a role of the MAOA as a modulator of neural plasticity related to cortical pain processing.
\end{abstract}

\section{Introduction}

Neural plasticity (i.e. the capacity of neurons to modify their function, chemical profile, or structure) plays a critical role in the cortical processing of repeated painful stimuli, which is dependent - among other factors - on the activity of brain-derived neurotrophic factor (Di

Correspondence: C. Di Lorenzo, as above.

E-mail: cherub@inwind.it

Received 2 September 2013, revised 15 November 2013, accepted 18 November 2013
Lorenzo et al., 2012a). One of the putative mechanisms through which brain-derived neurotrophic factor exerts its action in neural plasticity is by modulating monoaminergic function (Ren-Patterson et al., 2005), which is critical in cortical pain processing. The notion that monoamines are pivotal drivers of pain modulation and play an important role in neural plasticity processes has been widely documented (Bannister et al., 2009; Tully \& Bolshakov, 2010; Lesch \& Waider, 2012; Tritsch \& Sabatini, 2012).

Monoaminergic activity is modulated by monoamine oxidase type A (MAOA), a mitochondrial outer membrane-bound flavoprotein 
that catalyzes the oxidative deamination of serotonin, norepinephrine, and dopamine (Edmondson et al., 2004).

Although the role of MAOA has mainly been investigated in the context of psychiatric research, the possible therapeutic effect of MAOA inhibitors on pain-related conditions has been documented in humans and animal models (Pirildar et al., 2003; Mathiesen et al., 2006; Mattia \& Coluzzi, 2007; Villarinho et al., 2013).

Monoamine oxidase type $\mathrm{A}$ is a nuclear gene, located on the $\mathrm{X}$ chromosome (Xp11.3), that is characterized by an allelic variant of length in the promoter region (upstream of the coding region), a 30bp repeated sequence present in $3,3.5,4$, or 5 copies $(3 \mathrm{R}, 3.5 \mathrm{R}, 4 \mathrm{R}$, and $5 \mathrm{R}$, respectively). Alleles $3.5 \mathrm{R}$ and $4 \mathrm{R}$ are considered predictive of higher enzymatic activity [high-activity MAOA (HAM)], being transcribed 2-10 times more efficiently than $3 \mathrm{R}$ or $5 \mathrm{R}$ [low-activity MAOA (LAM)], suggesting an optimal length for the regulatory region (Sabol et al., 1998). This polymorphism is currently referred to as the MAOA upstream Variable Number Tandem Repeat (MAOA-uVNTR) region. An influence of genes regulating monoamine activity, mainly the MAOA gene, on pain sensitivity and tolerance to pain has been reported in humans (Kim et al., 2006; Treister et al., 2009). However, the influence of MAOA-uVNTR on painrelated conditions or on neural plasticity has been sparsely investigated. Recently, electrophysiological techniques, such as the trigeminal electric pain-related evoked potential (tPREP), have been used successfully to explore the neural correlates of cortical pain processing (Di Lorenzo et al., 2012a). The aim of the present study was to investigate the role of MAOA-uVNTR polymorphism on cortical pain processing by measuring the brain response to repetitive trigeminal electrical stimulation and its variations over time in healthy individuals. We hypothesized that cortical pain processing in response to repetitive trigeminal electrical stimulation in healthy individuals could be modulated by genetically determined MAOA activity.

\section{Materials and methods}

\section{Subjects}

Healthy adults between 20 and 40 years of age were recruited through an intranet advertisement among the staff of "Tor Vergata" University. Eligibility criteria were right-handedness, graduate education and no personal or family history of headache, any other chronic pain condition or neuropsychiatric disorders. Exclusion criteria were as follows:

1 The presence of uVNTR heterozygosis in women, due to the lack of reliable data for this group on MAOA activity at neuronal level (Meyer-Lindenberg et al., 2006). As MAOA is an X-linked gene, men are hemizygous carriers of either a high MAOA activity allele or a low MAOA activity allele. Women, however, carrying two alleles, can be heterozygous.

2 Use of any central nervous system-acting drugs in the last 6 months or analgesic in the $72 \mathrm{~h}$ prior to the experimental procedure.

Participants were asked not to drink coffee, tea or any other beverage containing stimulants and to refrain from smoking cigarettes in the $2 \mathrm{~h}$ before the beginning of the recording session. Information on the quality of sleep during the night prior to the recording was collected and the experiment was postponed if the subject reported a non-restoring sleep. Women were recorded during the follicular phase of the menstrual cycle. Written informed consent was obtained from all participants prior to entering the study, which received approval from the "Tor Vergata" University ethics commit- tee. All experimental procedures were carried out in accordance with the 'Ethical Principles for Medical Research Involving Human Subjects' (Declaration of Helsinki, 1964).

\section{Genotyping}

We obtained peripheral blood from all subjects and genomic DNA was extracted using standard procedures. Subjects were genotyped by restriction fragment length polymorphism analysis, according to the method described by Sabol et al. (1998) and previously adopted by our group (Frazzetto et al., 2007). Polymerase chain reaction was carried out using the following conditions: initial 5-min denaturing step at $95{ }^{\circ} \mathrm{C}$ followed by 35 cycles of $94{ }^{\circ} \mathrm{C}$ for $1 \mathrm{~min}$, $53.8{ }^{\circ} \mathrm{C}$ for $1 \mathrm{~min}$ and $72{ }^{\circ} \mathrm{C}$ for $1.5 \mathrm{~min}$, and a final extension phase of $72{ }^{\circ} \mathrm{C}$ for $10 \mathrm{~min}$. We used the following primer sequences: forward, 5'-ACAGCCTGACCGTG GAGAAG-3' and reverse, 5'-GAACGGACGCTCCATTCGGA-3'. Reactions were performed in a total volume of $25 \mu \mathrm{L}$ with $50 \mathrm{ng}$ genomic DNA, $1.5 \mathrm{~mm} \mathrm{MgCl}_{2}, 10 \mathrm{pmol}$ of each primer, $0.33 \mathrm{~mm} \mathrm{dNTPs}$, and $1.5 \mathrm{U}$ of native Taq (Promega, Madison, WI, USA). Polymerase chain reaction products were assayed on a $3 \%$ agarose gel. The primers used yielded 320-, 335-, 350- and 380-bp fragments corresponding to the 3-, 3.5-, 4- and 5-repeat alleles, respectively (Sabol et al., 1998). According to their genotype, subjects were classified as LAM (3- or 5-repeat form) and HAM (3.5- or 4-repeat form). To ensure that researchers were blinded at the time of neurophysiological data analysis, the results of genetic analysis were not made available prior to the analysis of neurophysiological data.

\section{Experimental setting}

\section{Stimulation}

Noxious stimuli were applied on the forehead (approximately $1 \mathrm{~cm}$ above the supraorbital foramen) to stimulate the supraorbital nerve (a terminal division of the trigeminal ophthalmic branch) using a custom-built planar concentric electrode (Bionen, Florence, Italy). The physical characteristics of the stimuli were consistent with those reported by Kaube et al. (2000) and were reported in detail in a previous study (Serrao et al., 2010). The duration of each stimulus was $100 \mu \mathrm{s}$. Individual thresholds for sensory and pain perception were defined as the minimum intensities of the electrical stimulus perceived as tactile and painful, respectively, determined by a sequence of three descending and ascending current intensities. The stimulation intensity was set at 1.5 -fold of the individual pain threshold. The recording session consisted of 31 stimuli of the same intensity with an inter-stimulus interval ranging between 14 and $16 \mathrm{~s}$. At the end of the recording session, subjects were asked to indicate the subjective pain intensity experienced during the electrical stimulation session by marking with a pencil a vertical line on a $100-\mathrm{mm}$ visual analogue scale, where 0 indicated 'no pain' and $100 \mathrm{~mm}$ indicated 'unbearable pain'.

\section{Electrophysiological recordings}

Subjects were seated in a reclining chair with a mounted headrest throughout the experiment. The recordings were performed using Ag/ $\mathrm{AgCl}$ disk electrodes (Bionen). According to published methodology, the active electrode was attached to the scalp on the $\mathrm{Cz}$ location of the International 10-20 System (Katsarava et al., 2006). To avoid distortions of the electroencephalogram due to asymmetrical impedances, signal was acquired on further active channels from the left (A1) and 
right (A2) earlobes, all electrodes being recorded to a common reference placed on the $\mathrm{Pz}$ position. The signal was off-line re-referenced to the computational A1 + A2 reference. A cephalic ground electrode was placed on Fpz. One polygraphic channel was used to monitor vertical and horizontal eye movements with the active electrode placed $1 \mathrm{~cm}$ lateral to the outer canthus of the right eye and the reference electrode placed on the lower eyelid. Electrode impedances were maintained below $5 \mathrm{k} \Omega$. The signal was amplified by a digital electroencephalography system (Galileo MIZAR-Sirius; EBNeuro, Florence, Italy) with a sampling rate of $2048 \mathrm{~Hz}$ and 24-bit resolution, and stored on a hard disk together with the signal of a digital trigger from the built-in electrical stimulator.

\section{Data analysis}

One of the investigators, who was blinded to the results of the genetic study, performed the analysis off-line. Digital data were exported to the European Data Format using NPX Lab 2010 (available at www.brainterface.com) and analyzed in the EEGLAB environment (http://www.sccn.ucsd.edu/eeglab/index.html) under MATLAB 7.7.0 R2010a (Mathworks Inc., Natick, MA, USA). The electroencephalography signal was digitally band-pass filtered between 1 and $100 \mathrm{~Hz}$ and segmented into $700-\mathrm{ms}$ epochs $(100 \mathrm{~ms}$ pre-stimulus to $600 \mathrm{~ms}$ post-stimulus). For each subject, the first epoch was rejected to avoid possible contamination by a startle response. The remaining 30 epochs were averaged in three sequential blocks of 10 responses and analyzed separately. We performed artifact rejection using the EEGLAB's semi-automatic procedure (Delorme et al., 2007). The electro-oculographic channel was used to further verify the results of the rejection method in the identification of ocular artifacts. The N2 and $\mathrm{P} 2$ components of the averaged evoked response were identified as the most negative and most positive peak, respectively, occurring between 90 and $260 \mathrm{~ms}$ after the stimulus. We measured the latency of the N2 and P2 components as well as the N2-P2 inter-peak amplitude for each block. We then computed the slope of the linear regression line (as expressed by the formula: $y=m x+b$ ) of the N2-P2 amplitudes over the three blocks in each subject to investigate changes over time of the evoked response amplitude. This derived measure of the evoked potential amplitude has the advantage of being relatively insensitive to inter-individual variability. A negative slope of the regression line was indicative of a decremental response, whereas a positive slope was an index of an incremental response.

\section{Sample size calculation}

An expert in biostatistics, who was not involved in the neurophysiological or genetic data collection, performed sample size calculation and statistical analysis (see the Statistical analysis section). As our primary endpoint was to detect differences in the slope of N2-P2 amplitude between HAM and LAM groups, a sample size calculation was based on pilot data from 20 subjects enrolled independently from the current study. Eleven were high and nine were low, with a slope index, respectively, of $-3.23 \pm 4.61$ and $0.81 \pm 4.73$. Assuming that the values in each subject group were normally distributed with a within-group SD of 4.99 , to fulfill a desired power of $90 \%$ with the significance level at $5 \%$, the required sample size was 67 subjects, 37 HAM and 30 LAM.

\section{Statistical analysis}

Due to the indices showing a non-normal distribution, we logarithmically transformed all of the variables to obtain a better approximation to a Gaussian curve, achieving an appropriate equivalence to a normal distribution (Kolmogorov-Smirnov test, $P>0.2$ ). ANOvA models were used to measure the differences among groups and between blocks and groups.

The differences in age, sensory threshold, pain threshold, visual analogue scale, grand averages of the N2 and P2 latencies, and N2P2 amplitude of the three blocks as well as in the slope index were analyzed using a two-way ANOvA (between-subject factors: 'gender', women vs. men; 'genotype', HAM vs. LAM). Gender was used as a between-subject factor due to evidence of gender differences in pain perception, sensitivity and tolerance (Mogil \& Bailey, 2010).

Repeated-measures ANOVA followed by univariate ANOVAs were performed on the N2-P2 measure to investigate the group effects (between-subject factors: 'gender' and 'genotype') on the three blocks (within-subjects factor: block 1 to block 3). Univariate results were analyzed only if the Wilks' Lambda multivariate significance criterion was achieved. The sphericity of the covariance matrix was verified with the Mauchly sphericity test. In the case of violation of the sphericity assumption, the Greenhouse-Geisser epsilon adjustment was used. Polynomial contrasts were constructed to analyze changes between blocks. As the data were divided into three blocks, only linear $(\mathrm{c} 1=0.707 \times$ block $1-0.707 \times$ block 3 , hereafter called 'slope') and quadratic (c2 $=0.408 \times$ block $1-0.816 \times$ block $2+0.408 \times$ block 3 ) contrasts could be defined and assessed for the main term (block effect in the whole sample) and for the 'block' $\times$ 'genotype' interaction (differences of block effects between low and high). Post-hoc tests were performed with Bonferroni's confidence interval adjustment for multiple comparisons to define which variables contributed to the major effects. The statistical significance was set at $P<0.05$.

\section{Results}

Sixty-seven participants [34 women, 33 men; age (mean \pm SD): $30.09 \pm 3.02$ and $29.85 \pm 4.20$ years, respectively] were enrolled in the study. Of these, 37 were genotyped as HAM and 30 as LAM. As MAOA promoter uVNTR polymorphism is X-linked and multiallelic, testing for Hardy-Weinberg equilibrium could be performed only in women (Philibert et al., 2008). According to the exclusion of heterozygosis in women, the Hardy-Weinberg equilibrium was not applicable to this sample.

The HAM and LAM groups did not differ significantly in gender, age, sensory threshold, pain threshold, or visual analogue scale (Table 1).

After the rejection of electroencephalography trials contaminated by artifacts, the number of epochs used for the analysis for the three blocks was (mean $\pm \mathrm{SD}$ ): $8.93 \pm 0.66,9.05 \pm 0.71$, and $9.02 \pm$ 0.71 , respectively. A repeated-measures ANOVA model showed no effect of 'block' (Wilks' Lambda $=0.98, F_{2,62}=0.82, P=0.45$ ), 'block' $\times$ 'genotype' (Wilks' Lambda $=0.999, F_{2,62}=0.02, P=$ 0.98 ), 'block' $\times$ 'gender' (Wilks' Lambda $=0.97, F_{2,62}=0.94$, $P=0.40$ ) and 'block' $\times$ 'genotype' $\times$ 'gender' (Wilks' Lambda $=$ $0.99, F_{2,62}=0.37, P=0.69$ ) interactions on the number of epochs left for the analysis.

\section{Amplitude and slope of the tPREP}

Two-way ANOva showed a significant effect of 'genotype' $\left(F_{1,63}=8.41, P=0.005\right)$ and 'gender' $\left(F_{1,63}=4.35, P=0.04\right)$ on the N2-P2 amplitude of the grand average of the three blocks; the 'genotype' $\times$ 'gender' interaction $\left(F_{1,63}=0.89, P=0.35\right)$ was not significant. The effect size, as measured by Cohen's f, was 0.35 for 
TABLE 1. Descriptive statistics of gender, age, sensory threshold (ST) and pain threshold (PT) in HAM and LAM

\begin{tabular}{llr}
\hline & HAM & LAM \\
\hline Gender* $^{*}$ & \\
Women & 20 & 14 \\
Men & 17 & 16 \\
Age (years) & $29.46 \pm 3.45$ & $30.60 \pm 3.80$ \\
ST (mA) & $1.73 \pm 0.65$ & $1.53 \pm 0.68$ \\
PT (mA) & $9.57 \pm 2.42$ & $9.23 \pm 2.62$ \\
VAS $^{\S}$ & $17.05 \pm 6.86$ & $17.33 \pm 6.93$ \\
\hline
\end{tabular}

Data are frequencies and mean $\pm \mathrm{SD}$. VAS, visual analogue scale. *Yates chi-square test: $\chi_{1}^{2}=0.13, P=0.72 .{ }^{\dagger}$ 'Genotype': $F_{1,63}=1.68, P=0.20$; 'gender': $F_{1,63}=0.09, \quad P=0.77 ;$ 'genotype' $\times$ 'gender': $F_{1,63}=0.42$, $P=0.52 .{ }^{\ddagger}$ Genotype': $\quad F_{1,63}=1.34, \quad P=0.25 ; \quad$ 'gender': $\quad F_{1,63}=0.12$, $P=0.73 ;$ 'genotype' $\times$ 'gender': $F_{1,63}=0.01, \quad P=0.94 .{ }^{\S}$ 'Genotype': $F_{1,63}=0.42, P=0.52$; 'gender': $F_{1,63}=1.89, P=0.17$; 'genotype' $\times$ 'gender': $F_{1,63}=0.20, P=0.66$. "'Genotype': $F_{1,63}=0.41, P=0.84$; 'gender': $F_{1,63}=0.20, P=0.65$; 'genotype' $\times$ 'gender': $F_{1,63}=0.67, P=0.42$.

'genotype' effect (Fig. 1A) and 0.26 for 'gender' effect, with values within Cohen's conventional values for medium (0.25) and large (0.40) effect. No significant differences were seen for the N2 ('genotype': $F_{1,63}=1.96, P=0.17$; 'gender': $F_{1,63}=1.69, \quad P=0.20$; 'genotype' $\times$ 'gender': $\left.F_{1,63}=0.15, P=0.70\right)$ and P2 latency ('genotype': $\quad F_{1,63}=1.02, \quad P=0.32 ; \quad$ 'gender': $\quad F_{1,63}=3.06$, $P=0.09$; 'genotype' $\times$ 'gender': $\left.F_{1,63}=0.15, P=0.70\right)$ of the grand average of the three blocks.

Multivariate tests of the repeated-measures ANOvA model for N2P2 amplitude revealed significant 'block' (Wilks' Lambda $=0.84$, $F_{2,62}=5.74, P=0.005$ ) and 'block' $\times$ 'genotype' effects (Wilks' Lambda $\left.=0.87, F_{2,62}=4.54, P=0.01\right)$ as represented in Fig. $1 \mathrm{~B}$ and $\mathrm{C}$; 'block' $\times$ 'gender' and 'block' $\times$ 'genotype' $\times$ 'gender' effects were not significant (Wilks' Lambda $=0.997, F_{2,62}=0.07$, $P=0.93$ and Wilks' Lambda $=0.996, \quad F_{2,62}=0.12, P=0.88$, respectively). After checking that the sphericity assumption was not violated (Mauchly's $\mathrm{W}=0.99, \chi_{2}^{2}=0.51, P=0.78$ ), univariate tests were performed. These showed significant 'block' $\left(F_{2,126}=6.35, \quad P=0.002\right)$ and 'block' $\times$ 'genotype' effects $\left(F_{2,126}=5.02, P<0.008\right)$ (Fig. 1B and C; Table 2), with effect size values ( $f=0.32$ and $f=0.28$, respectively) within Cohen's conventional values for medium $(0.25)$ and large $(0.40)$ effect. Polynomial contrasts of the three blocks allowed the assessment of the linear and quadratic components of the 'block' main effect and 'block' $x$ 'genotype' interaction term. In both cases, the $P$-values for the quadratic component were not significant $\left(F_{1,63}=0.62, P=0.43\right.$ and $F_{1,63}=0.23, P=0.63$, respectively), whereas the linear component (slope) was significant for the main term $\left(F_{1,63}=11.23, P=0.001\right)$ and for the interaction $\left(F_{1,63}=9.10, P<0.004\right)$. These findings indicate that the linear decrease observed in the whole sample was significantly different in the HAM and LAM groups. A post-hoc test on the 'block' main term (Table 2) indicated that the variables with the largest contribution to the effect were first block vs. third block $(P=0.0005)$, with an effect size, as measured by Cohen's d, of 0.31 , a value within Cohen's conventional values for small $(0.20)$ and medium (0.50) effect. Post-hoc analysis of the 'block' $\times$ 'genotype' interaction term (Fig. 1B; Table 2) indicated that the variables with the largest contribution to the effect in the HAM group were first block vs. third block $(P<0.0001$, Cohen's $\mathrm{d}=0.55)$ and, in the first block, HAM vs. LAM $(P<0.003$, Cohen's $\mathrm{d}=1.04)$. According to conventional values of Cohen's d, the effect size was considered medium and large, respectively.

Furthermore, two-way ANOva showed a significant 'genotype' effect $\left(F_{1,63}=14.76, P<0.0002\right)$ on the slope index, accounting for a large effect size $(f=0.48)$ (Fig. 1D); 'gender' effect $\left(F_{1,63}=0.02, P=0.90\right)$ and 'genotype' $\times$ 'gender' interaction effect $\left(F_{1,63}=0.29, P=0.59\right)$ were not significant.

The slope was significantly different from 0 in the HAM group $[-3.67,95 \%$ confidence interval $(-4.83,-2.51)]$ but not in the LAM group $[0.07,95 \%$ confidence interval (-1.57, 1.71)] (Fig. 1D). The significant difference between slope index means was also confirmed when tested by means of Welch's robust test for
A

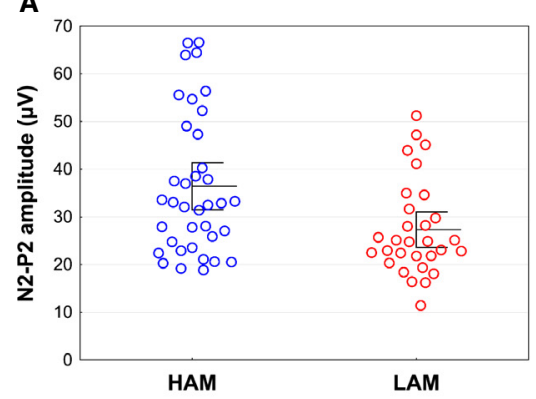

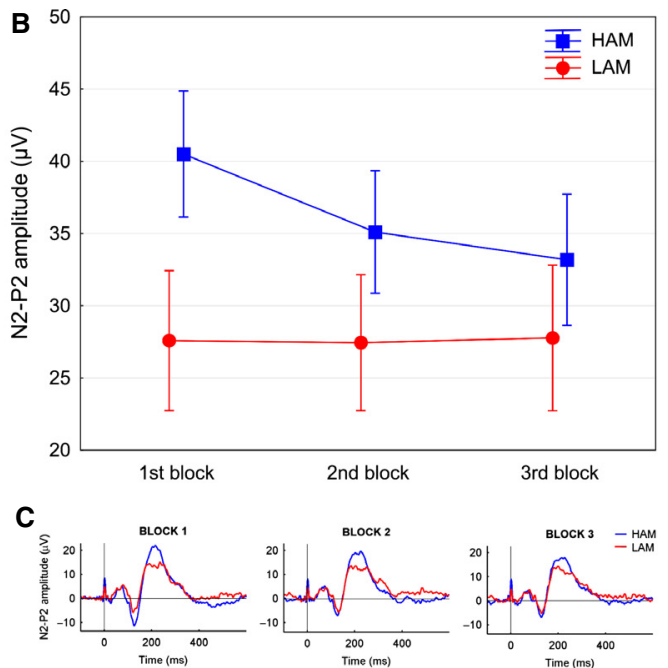

D

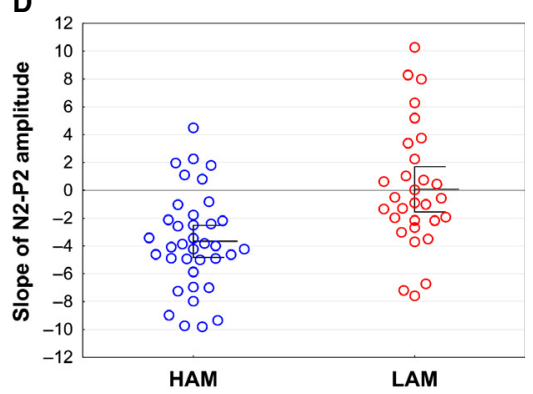

FIG. 1. (A) tPREP N2-P2 amplitudes of the three-block grand average of HAM (left panel) and LAM (right panel). Each circle represents one participant. Mean and dispersion around the mean are rendered with the middle line indicating the mean value and the vertical bar indicating the $95 \%$ confidence interval. (B) N2-P2 amplitudes of HAM (blue line) and LAM (red line) in the three blocks. (C) Grand average tPREP of HAM (blue line) and LAM (red line) in the three blocks. (D) Slope values of the N2-P2 amplitude of the tPREP in HAM (left panel) and LAM (right panel). Each circle represents one participant. Mean and dispersion around the mean are rendered as in (A). 
TABLE 2. Indices of tPREP in whole sample, HAM and LAM

\begin{tabular}{lccc}
\hline & Whole sample & HAM & LAM \\
\hline N2 latency (ms) & & & \\
First block & $127.96 \pm 10.82$ & $125.96 \pm 12.00$ & $130.42 \pm 8.73$ \\
Second block & $129.58 \pm 14.20$ & $128.34 \pm 13.55$ & $131.12 \pm 15.06$ \\
Third block & $129.91 \pm 16.75$ & $127.51 \pm 14.47$ & $132.86 \pm 19.03$ \\
Grand average & $129.15 \pm 12.06$ & $127.27 \pm 10.95$ & $131.47 \pm 13.13$ \\
& & & \\
P2 latency (ms) & & & \\
First block & $208.69 \pm 26.85$ & $211.11 \pm 23.01$ & $205.70 \pm 31.10$ \\
Second block & $207.62 \pm 25.49$ & $210.75 \pm 23.47$ & $203.75 \pm 27.69$ \\
Third block & $207.14 \pm 26.06$ & $209.90 \pm 24.60$ & $203.75 \pm 27.78$ \\
Grand average & $208.06 \pm 26.64$ & $210.41 \pm 24.23$ & $205.18 \pm 29.50$ \\
& & & \\
N2-P2 amplitude $(\mu \mathrm{V})$ & & & \\
First block & $34.70 \pm 14.90^{*}$ & $40.67 \pm 15.66^{*}{ }^{\dagger}$ & $27.32 \pm 9.94^{\dagger}$ \\
Second block & $31.64 \pm 13.63 *$ & $35.27 \pm 14.45^{*}$ & $27.14 \pm 11.22$ \\
Third block & $30.70 \pm 14.19^{*}$ & $33.33 \pm 15.70^{*}$ & $27.46 \pm 11.50$ \\
Grand average & $32.35 \pm 13.50$ & $36.43 \pm 14.73^{\dagger}$ & $27.31 \pm 9.91^{\dagger}$ \\
Slope of N2-P2 & $-2.00 \pm 4.31$ & $-3.67 \pm 3.48$ & $0.07 \pm 5.01$ \\
blocks & & & \\
& & &
\end{tabular}

Data, shown as mean $\pm \mathrm{SD}$, are raw values. ${ }^{*}{ }^{\dagger}$ Significant difference among blocks and between HAM and LAM, respectively (see Results for details).

equality of means $(P<0.0004)$. Despite the Levene's test for homogeneity of variance not being significant $(P=0.29)$, this procedure was applied due to the high heterogeneity of the LAM group. As shown in Fig. 1D, the LAM subjects' heterogeneity was related to the coexistence of subjects with a clear N2-P2 decrease (similar to HAM subjects) and of subjects with null or even positive slope.

Finally, the significance of the 'block' $\times$ 'genotype' interaction and of its linear component was reliably confirmed even after entering the N2-P2 amplitude of the first block as covariate.

\section{Latency of the TPREP}

The N2 and P2 latency did not change significantly over the three blocks (multivariate tests, $P$-values of repeated-measures ANOvA models: N2, 'block', $P=0.46$, 'block' $\times$ 'genotype', $P=0.65$, 'block' $\times$ 'gender', $P=0.07$, 'block' $\times$ 'genotype' $\times$ 'gender', $P=0.65$; P2, 'block', $P=0.82$, 'block' $\times$ 'genotype', $P=0.88$, 'block' $\times$ 'gender', $P=0.06$, 'block' $\times$ 'genotype' $\times$ 'gender', $P=0.59$ ).

\section{Discussion}

Consistent with our hypothesis, the two allelic variants of the MAOA genes associated with high and low enzymatic activity showed different cortical pain processing in response to repetitive trigeminal electrical stimulation.

The amplitude of evoked potentials is a reliable quantitative measure of the extent of neural activation in response to external stimuli (Miltner et al., 1987) and is regarded as a measure of brain plastic adaptation (Delvendahl et al., 2012). The N2-P2 cortical response to trigeminal painful electrical stimulation is sustained by the anterior cingulate cortex (Obermann et al., 2009) and encodes cognitive and evaluative aspects of stimulus processing, representing an indirect readout of central nociceptive networks (Apkarian et al., 2005; Duric \& McCarson, 2006).

As expected for healthy adults, we observed a habituation phenomenon, i.e. a progressive reduction of the N2-P2 amplitude to repeated electrical noxious stimuli in the whole sample. Electrical stimulation of cutaneous afferents is associated with long-term depression of nociception, expression of plastic changes in human pain processing (Rottmann et al., 2008). Similarly, the response decrement observed in our study after repeated stimulations could be a manifestation of short- and long-term neural plasticity, mediated by pre- or post-synaptic modulation, and interpreted as a form of learning aimed at protecting the brain from painful overstimulation and synaptic exhaustion (Coppola et al., 2013).

When we analyze the two groups separately, carriers of the LAM genotype showed a significantly lower amplitude of the grand-averaged N2-P2 compared with HAM subjects (Fig. 1A), with the expected degree of overlap between the two groups given the noncategorical distribution of MAOA activity. Differences in N2-P2 amplitude between the HAM and LAM groups could result from a lesser degree of cortical processing of painful stimuli in LAM subjects and the expression of impaired neural plasticity and reduced ability to respond to the painful stimulus. Although a relationship between monoaminergic activity and indices of cortical processing has already been reported for other stimulus modalities, to the best of our knowledge this is the first observation about monoaminergic influences on tPREP. Studies in healthy individuals (Strobel et al., 2003; Simmons et al., 2011) and animals (Adler et al., 1988; Manjarrez et al., 2005; Siegel et al., 2005) have reported a modulating effect of monoaminergic activity on the loudness dependence of the auditory evoked potential. Furthermore, dopaminergic stimulation modulates the motor evoked potential amplitude in patients with restless legs syndrome, chronic stroke and Alzheimer's disease (Acler et al., 2009; Scalise et al., 2010; Martorana et al., 2013). Moreover, in patients with Parkinson's disease, dopaminergic stimulation has shown facilitating effects on the transcranial direct current stimulation-induced amplitude reduction of laser-evoked potentials. The effect of transcranial direct current stimulation on the cortex is mediated by neural plastic modifications and thus dopaminergic stimulation seems to aid neural plasticity in patients with Parkinson's disease (Terney et al., 2008). Our observation is in line with the above-mentioned findings and reinforces the suggestion that monoaminergic activity (expressed indirectly in our study by the MAOA polymorphisms) can modulate the degree of cortical responses to a wide range of stimuli.

The analysis of the amplitude of the tPREP by blocks allowed tracking of the time-course of the cortical response during progressive exposure to repetitive pain stimulation. We found a significant difference between the two MAOA genotype groups in the amplitude of the first block of responses. LAM carriers showed a lower amplitude of the first block compared with HAM carriers (Fig. 1B). The first block is regarded as a measure of sensitization (Thompson \& Spencer, 1966; Groves \& Thompson, 1970). It represents the baseline level of arousal and is determined by a mechanism not dissimilar from short-term potentiation, i.e. a facilitation of neurotransmission that occurs at the beginning of the stimulus session that accounts for the initial transient increase in response amplitude. In the pain network, which is a crucial substrate of the tPREP response, central sensitization is a measure of neural excitability (Woolf \& Wall, 1986). In our study, the observed lower sensitization in LAM compared with HAM subjects may therefore reflect differences in baseline cortical excitability between the two groups. Our findings are consistent with the observations that monoamine oxidases modulates sensitization in animals (Villégier et al., 2003). The sensitization process involves a pre-synaptic facilitation mediated by serotonergic transmission, originating from a group of facilitating interneurons (Frost et al., 1985; Bergold et al., 1990; Braha et al., 1990). We could speculate that the LAM genotype affects 
pain-related cortical excitability mainly by an abnormal serotoninergic modulation.

Block analysis of tPREP also allowed the evaluation of genetically determined differences in behavior of the response to repeated stimulation. In our study, although a reduction in the amplitude of the response for consecutive blocks is observed as expected in the HAM group and in the whole sample, it was not seen in LAM individuals. The progressive reduction of response to trigeminal painful repetitive stimulation, observed in the whole group and in HAM subjects, is a habituation phenomenon. Habituation is the simplest form of learning and, as mentioned above, is a protective plastic adaptation of the cerebral cortex to repeated stimuli. Habituation is a process common to all sensory modalities (Thompson \& Spencer, 1966; Groves \& Thompson, 1970) and can be elicited in response to repetitive nociceptive stimuli (Milne et al., 1991). Habituation phenomena in brain structures of the so-called 'pain matrix' (Iannetti \& Mouraux, 2010) have been documented with functional neuroimaging studies using a wide range of painful stimulations both within a single experimental session (Becerra et al., 1999; Ibinson et al., 2004; Petrovic et al., 2004; Christmann et al., 2007; Mobascher et al., 2010) and over repeated sessions on different days (Bingel et al., 2007, 2008). Habituation to pain results from a balance between central nociception and antinociception (Bingel et al., 2007, 2008; Rennefeld et al., 2010).

Although the central mechanisms of neural plasticity involved in habituation to painful stimuli are unknown, a possible interpretation of our findings relates to the effect of MAOA-uVNTR on the plastic adaptation of brain structures to pain processing. The progressive reduction in N2-P2 amplitude over the three blocks observed in HAM subjects could be induced by cortical plastic modification in the pain matrix (see below) structures or in the brainstem monoaminergic nuclei. These structures are central effectors of the endogenous pain control system and play a crucial role in the central processing of sensory stimuli, by controlling the signal-to-noise ratio in cortical and thalamo-cortical neurons (Mesulam, 1990). The impaired monoaminergic modulation in the LAM genotype could affect these plastic modifications (Belsky et al., 2009), resulting in the lack of decremental response to painful repeated stimulation.

The emerging role of the MAOA gene in the neural plasticity of electrophysiological responses is not an isolated case. The angiotensin-converting enzyme Insertion/Deletion polymorphism, not strictly related to the production of neurotrophins, has been associated with plastic modifications across blocks of evoked potentials in a group of chronic migraine patients with medication overuse (Di Lorenzo et al., 2012b).

In the present study, MAOA-uVNTR polymorphism influenced multiple measures of the tPREP, i.e. amplitude, sensitization and habituation in response to repeated stimulation. The thalamus, anterior cingulate cortex, insula, and primary and secondary somatosensory cortices constitute the pain matrix and MAOA is expressed in most of these brain structures. Through its enzymatic activity, MAOA exerts a plastic effect, genetically determined by MAOAuVNTR, resulting in differences between the high- and low-activity allelic forms in neural excitability and volume of nuclei involved in pain and emotional processing (Meyer-Lindenberg et al., 2006).

Finally, based on our findings, we suggest that MAOA-uVNTR appears to exert an influence on tPREP responses influencing monaminergic enzymatic activity. The monoaminergic system is crucial for tuning cortical excitability including sensitization and habituation processes (Leussis \& Bolivar, 2006). N2-P2 cortical responses can therefore be considered as a reliable neurophysiological correlate of the synaptic plasticity attributed to MAOA activity. Our findings suggest a role of MAOA as pain neuromodulator through mechanisms involving neural plasticity.

\section{Acknowledgements}

This work was supported by the Don Gnocchi Onlus Foundation (grant number 08RC02) (Ricerca Corrente 2011). Pain research at the Aston Brain Centre is supported by the Dr Hadwen and Lord Dowding Trusts for Humane Research and by the Wellcome Trust.

\section{Abbreviations}

HAM, high-activity monoamine oxidase type A; LAM, low-activity monoamine oxidase type A; MAOA, monoamine oxidase type A; MAOA-uVNTR, MAOA upstream Variable Number Tandem Repeat.

\section{References}

Acler, M., Fiaschi, A. \& Manganotti, P. (2009) Long-term levodopa administration in chronic stroke patients. A clinical and neurophysiologic singleblind placebo-controlled cross-over pilot study. Restor. Neurol. Neuros., 27, 277-283

Adler, L.E., Pang, K., Gerhardt, G. \& Rose, G.M. (1988) Modulation of the gating of auditory evoked potentials by norepinephrine: pharmacological evidence obtained using a selective neurotoxin. Biol. Psychiat., 24, 179190.

Apkarian, A.V., Bushnell, M.C., Treede, R.D. \& Zubieta, J.K. (2005) Human brain mechanisms of pain perception and regulation in health and disease. Eur. J. Pain, 9, 463-484.

Bannister, K., Bee, L.A. \& Dickenson, A.H. (2009) Preclinical and early clinical investigations related to monoaminergic pain modulation. Neurotherapeutics, 6, 703-712.

Becerra, L.R., Breiter, H.C., Stojanovic, M., Fishman, S., Edwards, A., Comite, A.R., Gonzalez, R.G. \& Borsook, D. (1999) Human brain activation under controlled thermal stimulation and habituation to noxious heat: an fMRI study. Magn. Reson. Med., 41, 1044-1057.

Belsky, J., Jonassaint, C., Pluess, M., Stanton, M., Brummett, B. \& Williams, R. (2009) Vulnerability genes or plasticity genes? Mol. Psychiatr., 14, 746-754.

Bergold, P.J., Sweatt, J.D., Winicov, I., Weiss, K.R., Kandel, E.R. \& Schwartz, J.H. (1990) Protein synthesis during acquisition of long-term facilitation is needed for the persistent loss of regulatory subunits of the Aplysia cAMPdependent protein kinase. Proc. Natl. Acad. Sci. USA, 87, 3788-3791.

Bingel, U., Schoell, E., Herken, W., Buchel, C. \& May, A. (2007) Habituation to painful stimulation involves the antinociceptive system. Pain, 131, 21-30.

Bingel, U., Herken, W., Teutsch, S. \& May, A. (2008) Habituation to painful stimulation involves the antinociceptive system - a 1-year follow-up of 10 participants. Pain, 140, 393-394.

Braha, O., Dale, N., Hochner, B., Klein, M., Abrams, T.W. \& Kandel, E.R. (1990) Second messenger involved in the two processes of presynaptic facilitation that contribute to sensitization and dishabituation in Aplysia sensory neurons. Proc. Natl. Acad. Sci. USA, 87, 2040-2044.

Christmann, C., Koeppe, C., Braus, D.F., Ruf, M. \& Flor, H. (2007) A simultaneous EEG-fMRI study of painful electric stimulation. Neurolmage, 34, 1428-1437.

Coppola, G., Di Lorenzo, C., Schoenen, J. \& Pierelli, F. (2013) Habituation and sensitization in primary headaches. J. Headache Pain, 14, 65.

Delorme, A., Sejnowski, T. \& Makeig, S. (2007) Enhanced detection of artifacts in EEG data using higher-order statistics and independent component analysis. NeuroImage, 34, 1443-1449.

Delvendahl, I., Jung, N.H., Kuhnke, N.G., Ziemann, U. \& Mall, V. (2012) Plasticity of motor threshold and motor-evoked potential amplitude - a model of intrinsic and synaptic plasticity in human motor cortex? Brain Stimul., 5, 586-593.

Di Lorenzo, C., Di Lorenzo, G., Daverio, A., Pasqualetti, P., Coppola, G., Giannoudas, I., Barone, Y., Grieco, G.S., Niolu, C., Pascale, E., Santorelli, F.M., Nicoletti, F., Pierelli, F., Siracusano, A. \& Seri, S. (2012a) The Val66Met polymorphism of the BDNF gene influences trigeminal painrelated evoked responses. J. Pain, 13, 866-873.

Di Lorenzo, C., Coppola, G., Currà, A., Grieco, G., Santorelli, F.M., Lepre, C., Porretta, E., Pascale, E. \& Pierelli, F. (2012b) Cortical response to somatosensory stimulation in medication overuse headache patients is 
influenced by angiotensin converting enzyme (ACE) I/D genetic polymorphism. Cephalalgia, 32, 1189-1197.

Duric, V. \& McCarson, K.E. (2006) Effects of analgesic or antidepressant drugs on pain- or stress-evoked hippocampal and spinal neurokinin-1 receptor and brain-derived neurotrophic factor gene expression in the rat. J. Pharmacol. Exp. Ther., 319, 1235-1243.

Edmondson, D.E., Mattevi, A., Binda, C., Li, M. \& Hubálek, F. (2004) Structure and mechanism of monoamine oxidase. Curr. Med. Chem., 11, 1983-1993.

Frazzetto, G., Di Lorenzo, G., Carola, V., Proietti, L., Sokolowska, E., Siracusano, A., Gross, C. \& Troisi, A. (2007) Early trauma and increased risk for physical aggression during adulthood: the moderating role of MAOA genotype. PLOS ONE, 2, e486.

Frost, W.N., Castelluci, V.F., Hawkins, R.D. \& Kandel, E.R. (1985) Monosynaptic connections made by the sensory neurons of the gill- and siphon-withdrawal reflex in Aplysia participate in the storage of long-term memory for sensitization. Proc. Natl. Acad. Sci. USA, 82, 8266-8269.

Iannetti, G.D. \& Mouraux, A. (2010) From the neuromatrix to the pain matrix (and back). Exp. Brain Res., 205, 1-12.

Groves, P.M. \& Thompson, R.F. (1970) Habituation: a dual-process theory. Psychol. Rev., 77, 419-450.

Ibinson, J.W., Small, R.H., Algaze, A., Roberts, C.J., Clark, D.L. \& Schmalbrock, P. (2004) Functional magnetic resonance imaging studies of pain: an investigation of signal decay during and across sessions. Anesthesiology, 101, 960-969.

Katsarava, Z., Ayzenberg, I., Sack, F., Limmroth, V., Diener, H.C. \& Kaube, H. (2006) A novel method of eliciting pain-related potentials by transcutaneous electrical stimulation. Headache, 46, 1511-1517.

Kaube, H., Katsarava, Z., Käufer, T., Diener, H. \& Ellrich, J. (2000) A new method to increase nociception specificity of the human blink reflex. Clin. Neurophysiol., 111, 413-416.

Kim, H., Lee, H., Rowan, J., Brahim, J. \& Dionne, R.A. (2006) Genetic polymorphisms in monoamine neurotransmitter systems show only weak association with acute post-surgical pain in humans. Mol. Pain, 2, 24.

Lesch, K.P. \& Waider, J. (2012) Serotonin in the modulation of neural plasticity and networks: implications for neurodevelopmental disorders. Neuron, 76, 175-191.

Leussis, M. \& Bolivar, V. (2006) Habituation in rodents: a review of behavior, neurobiology, and genetics. Neurosci. Biobehav. R., 30, 1045-1064.

Manjarrez, G., Hernandez, E., Robles, A. \& Hernandez, J. (2005) N1/P2 component of auditory evoked potential reflect changes of the brain serotonin biosynthesis in rats. Nutr. Neurosci., 8, 213-218.

Martorana, A., Di Lorenzo, F., Esposito, Z., Lo Giudice, T., Bernardi, G., Caltagirone, C. \& Koch, G. (2013) Dopamine $\mathrm{D}_{2}$-agonist Rotigotine effects on cortical excitability and central cholinergic transmission in Alzheimer's disease patients. Neuropharmacology, 64, 108-113.

Mathiesen, O., Imbimbo, B.P., Hilsted, K.L., Fabbri, L. \& Dahl, J.B. (2006) CHF3381, a N-methyl-D-aspartate receptor antagonist and monoamine oxidase-A inhibitor, attenuates secondary hyperalgesia in a human pain model. J. Pain, 7, 565-574.

Mattia, C. \& Coluzzi, F. (2007) Indantadol, a novel NMDA antagonist and nonselective MAO inhibitor for the potential treatment of neuropathic pain. IDrugs, 10, 636-644

Mesulam, M.M. (1990) Large-scale neurocognitive networks and distributed processing for attention, language, and memory. Ann. Neurol., 28, 597-613.

Meyer-Lindenberg, A., Buckholtz, J.W., Kolachana, B., Hariri, A.R., Pezawas, L., Blasi, G., Wabnitz, A., Honea, R., Verchinski, B., Callicott, J.H., Egan, M., Mattay, V. \& Weinberger, D.R. (2006) Neural mechanisms of genetic risk for impulsivity and violence in humans. Proc. Natl. Acad. Sci. USA, 103, 6269-6274.

Milne, R.J., Kay, N.E. \& Irwin, R.J. (1991) Habituation to repeated painful and non-painful cutaneous stimuli: a quantitative psychophysical study. Exp. Brain Res., 87, 438-444.

Miltner, W., Larbig, W. \& Braun, C. (1987) Habituation of subjective ratings and event-related potentials to painful intracutaneous electrical stimulation of the skin. J. Psychophysiol., 1, 221-228.

Mobascher, A., Brinkmeyer, J., Warbrick, T., Musso, F., Schlemper, V., Wittsack, H.J., Saleh, A., Schnitzler, A. \& Winterer, G. (2010) Brain activation patterns underlying fast habituation to painful laser stimuli. Int. J. Psychophysiol., 75, 16-24.
Mogil, J.S. \& Bailey, A.L. (2010) Sex and gender differences in pain and analgesia. Prog. Brain Res., 186, 141-157.

Obermann, M., Pleger, B., de Greiff, A., Stude, P., Kaube, H., Diener, H.C. \& Katsarava, Z. (2009) Temporal summation of trigeminal pain in human anterior cingulate cortex. Neurolmage, 46, 193-200.

Petrovic, P., Petersson, K.M., Hansson, P. \& Ingvar, M. (2004) Brainstem involvement in the initial response to pain. Neurolmage, 22, 995-1005.

Philibert, R.A., Gunter, T.D., Beach, S.R., Brody, G.H. \& Madan, A. (2008) MAOA methylation is associated with nicotine and alcohol dependence in women. Am. J. Med. Genet. B., 147B., 565-570.

Pirildar, S., Sezgin, U., Elbi, H., Uyar, M. \& Zileli, B. (2003) A preliminary open-label study of moclobemide treatment of pain disorder. Psychopharmacol. Bull., 37, 127-134.

Rennefeld, C., Wiech, K., Schoell, E.D., Lorenz, J. \& Bingel, U. (2010) Habituation to pain: further support for a central component. Pain, 148, 503-508.

Ren-Patterson, R.F., Cochran, L.W., Holmes, A., Sherrill, S., Huang, S.J., Tolliver, T., Lesch, K.P., Lu, B. \& Murphy, D.L. (2005) Loss of brainderived neurotrophic factor gene allele exacerbates brain monoamine deficiencies and increases stress abnormalities of serotonin transporter knockout mice. J. Neurosci. Res., 79, 756-771.

Rottmann, S., Jung, K. \& Ellrich, J. (2008) Electrical low-frequency stimulation induces homotopic long-term depression of nociception and pain from hand in man. Clin. Neurophysiol., 119, 1895-1904.

Sabol, S.Z., Hu, S. \& Hamer, D. (1998) A functional polymorphism in the monoamine oxidase A gene promoter. Hum. Genet., 103, 273-279.

Scalise, A., Pittaro-Cadore, I., Janes, F., Marinig, R. \& Gigli, G.L. (2010) Changes of cortical excitability after dopaminergic treatment in restless legs syndrome. Sleep Med., 11, 75-81.

Serrao, M. Coppola, G., Di Lorenzo, C., Di Fabio, R., Padua, L., Sandrini, G. \& Pierelli, F. (2010) Nociceptive trigeminocervical reflexes in healthy subjects. Clin. Neurophysiol., 121, 1563-1568.

Siegel, S.J., Maxwell, C.R., Majumdar, S., Trief, D.F., Lerman, C., Gur, R.E., Kanes, S.J. \& Liang, Y. (2005) Monoamine reuptake inhibition and nicotine receptor antagonism reduce amplitude and gating of auditory evoked potentials. Neuroscience, 133, 729-738.

Simmons, J.G., Nathan, P.J., Berger, G. \& Allen, N.B. (2011) Chronic modulation of serotonergic neurotransmission with sertraline attenuates the loudness dependence of the auditory evoked potential in healthy participants. Psychopharmacology, 217, 101-110.

Strobel, A., Debener, S., Schmidt, D., Hünnerkopf, R., Lesch, K.P. \& Brocke, B. (2003) Allelic variation in serotonin transporter function associated with the intensity dependence of the auditory evoked potential. Am. J. Med. Genet. B., 118B, 41-47.

Terney, D., Bergmann, I., Poreisz, C., Chaieb, L., Boros, K., Nitsche, M.A., Paulus, W. \& Antal, A. (2008) Pergolide increases the efficacy of cathodal direct current stimulation to reduce the amplitude of laser-evoked potentials in humans. J. Pain Symptom Manag., 36, 79-91.

Thompson, R.F. \& Spencer, W.A. (1966) Habituation: a model phenomenon for the study of neuronal substrates of behavior. Psychol. Rev., 73, $16-43$.

Treister, R., Pud, D., Ebstein, R.P., Laiba, E., Gershon, E., Haddad, M. \& Eisenberg, E. (2009) Associations between polymorphisms in dopamine neurotransmitter pathway genes and pain response in healthy humans. Pain, 147, 187-193.

Tritsch, N.X. \& Sabatini, B.L. (2012) Dopaminergic modulation of synaptic transmission in cortex and striatum. Neuron, 76, 33-50.

Tully, K. \& Bolshakov, V.Y. (2010) Emotional enhancement of memory: how norepinephrine enables synaptic plasticity. Mol. Brain, 3, 15.

Villarinho, J.G., de Vargos Pinherio, K., de Vargas Pinheiro, F., Oliveira, S.M., Machado, P., Martins, M.A., Bonacorso, H.G., Zanatta, N., Fachinetto, R. \& Ferreira, J. (2013) The antinociceptive effect of reversible monoamine oxidase-A inhibitors in a mouse neuropathic pain model. Prog. Neuro-Psychoph., 44, 136-142.

Villégier, A.S., Blanc, G., Glowinski, J. \& Tassin, J.P. (2003) Transient behavioral sensitization to nicotine becomes long-lasting with monoamine oxidases inhibitors. Pharmacol. Biochem. Be., 76, 267-274.

Woolf, C.J. \& Wall, P.D. (1986) Relative effectiveness of C primary afferent fibers of different origins in evoking a prolonged facilitation of the flexor reflex in the rat. J. Neurosci., 6, 1433-1442. 